\title{
Psychological Impact of Acne Vulgaris in Basic Science Medical Students in Nepal
}

\author{
Aryal E1, Shrestha $\mathbf{S}^{1}$, Shrestha $\mathbf{P}^{2}$, Pokhrel $\mathbf{G}^{3}$, Bhattarai $\mathbf{S}^{3}$ \\ ${ }^{1}$ Assistant Professor, ${ }^{2}$ Lecturer, ${ }^{3}$ Associate Professor, Department of Dermatology, Kathmandu Medical College
}

\begin{abstract}
Introduction: Acne vulgaris is a disorder of the sebaceous gland affecting mostly the adolescent age. Although acne does not cause direct physical impairment, it can produce significant psychosocial stress. Due to the paucity of data, there is a poor understanding of etiopathogenesis, treatment and psycho-social impact of acne in the Nepalese population. This study is the first of its kind in Nepal.

Objectives: To determine the beliefs, knowledge, misconceptions, myth, psycho-social impact about acne in recently joined undergraduate medical students attending basic science.

Materials and Methods: A cross- sectional study was conducted in two medical colleges in Kathmandu affiliated to Kathmandu University (KU), which followed the same curriculum. Medical students of basic science were enrolled in the study. Self-administered questionnaire was used to collect the data regarding their belief and perception about acne.

Results: A total of 247 students constituted the study population and female to male ratio was 1:1.62. Stress, oily skin, poor hygiene were the most common causative factors for acne. Belief and perception of acne included bad blood, menstrual cycle, eve teasing, evil eye. According to them, the best way to treat acne was herbal remedies. They liked to explain acneas being related to puberty, followed by dirty face. Psychological impact of acne was embarrassment followed by lack of confidence and impaired social contact. Suicidal tendency due to acne was found in 3.6\%.

Conclusion: Misconception about acne are widespread and enduring among basic science medical students. Health education program on acne is needed to improve their condition.
\end{abstract}

Key words: Acne vulgaris; health education; sebaceous gland; students, medical; surveys and questionnaires

\section{Introduction}

A cne vulgaris is a disorder of sebaceous gland affecting mostly the adolescent age group with prevalence of $91 \%$ in males and $79 \%$ females. ${ }^{1}$ Similarly, in medical students it varies from $56 \%$ to $62 \% .^{2}$ Although acne does not cause direct physical impairment, it can produce significant psychosocial stress. There are many researches on the etiopathogenesis, treatment and psycho-social impact of acne among patients outside Nepal, but there is a poor understanding of this in the Nepalese population due to the paucity of data available. Misconceptions exist in the community regarding factors that can exacerbate and progress acne vulgaris. Thus, taking this into consideration, the purpose of our present study

Address of Correspondence:

Dr. Eliz Aryal

Assistant Professor

Kathmandu Medical College, Sinamangal, Nepal.

E-mail: eliz_aryal@yahoo.com was to determine the beliefs and misconceptions, myth about acne in recently joinedbasic science medical students. The main objectives of our study were to assess the belief, knowledge, misconception and psycho-social impact of acne vulgaris in basic science undergraduate medical students in their medical career.

Submitted: $24^{\text {th }}$ October 2017

Accepted: $24^{\text {th }}$ January 2018

Published: $21^{\text {st }}$ March 2018

How to cite this article

Aryal E, Shrestha S, Shrestha P, Pokhrel G, Bhattarai S. Psychological impact of acne vulgaris in basic science medical students in Nepal. Nepal Journal of Dermatology Venereology and Leprology. 2018;16(1):30-4. doi: http://dx.doi.org/10.3126/ njdvl.v16i1.19401

\section{(c) (i)}

Licensed under CC BY 4.0 International License which permits use, distribution and reproduction in any medium, provided the original work is properly cited. 


\section{Materials and Methods}

A cross-sectional study was conducted in two medical colleges in Kathmandu, affiliated to Kathmandu University (KU) at Kathmandu Medical College Teaching Hospital, Sinamangal and Kathmandu University teaching hospital, Dhulikhel. The study was conducted between April 2015 to May 2016 after ethical clearance in 2015 March. According to KU schedule, dermatology lecture and clinical posting is only conducted in third and fourth year of medical schooling, hence we enrolled the medical students of basic science (first and second years). A pilot study was conducted in first 20 students to check the understanding and clarity of questionnaires. Based on the results, questions were made to avoid confusion to make easier understanding and interpretation by students. Students who had a history of acne in thepast or present were included in the study. Before the distribution of the questionnaire, a verbal consent was obtained from each student. The students completed the questionnaire during regular lecture class (teaching time) without discussion with peer partners.

The questionnaires were divided in four parts: 1) socialdemographic data 2) causative factor 3) knowledge and beliefs about acne and 4) psychological effects of acne in theirmedical career. The identification of student was not disclosed, only the data collected from the questionnaire were used.

Data entry and descriptive analysis were done using SPSS version 16.0 for windows.

\section{Results}

A total of 247 students with known cases of acne inthepast and present constituted the study population. The mean age was $20.05 \pm 1.107$ years. Age of the students ranged from 18 years to 23 years. The female to male ratio was 1:1.62. Onset of acne was most common in student of age group 15-20 years (48.2\%) (Table:1). Positive family history was present in $74(30 \%)$ students.

Opinion about causative factors for acne:

The second part of the questionnaire pertained to the causative factors of acne. They responded as acne being a contagious disease (2.8\%), was caused by a bad thought $(6.1 \%)$, frequent washing of face $(10.5 \%)$, family history of pimple (10.5\%), poor hygiene (33.2\%), diet (oily food, chocolate, cold drink) (38.9\%), stress
$(44.9 \%)$, puberty related condition $(58.3 \%)$, oily skin (64.8\%) and cosmetic use (26.7\%) (Table.2).

Opinion about belief and perception of acne:

The third part of the questionnaire pertained to opinion about belief and perception of acne. They believed that acne was due to evil eye (11.3\%), eve teasing (21.9\%), bad blood (46.2\%), menstrual cycle (71.3\%). Around $83 \%$ believed that there was a role of medication in the treatmentof acne and similarly, $87 \%$ thought that acne could be cured (Figure 1). When means of treatment for acne was asked to the students, only $16.2 \%$ chose Allopathic medicines (Table 3).

The second part of questionnaire was about attitude towards acne. "As a medical student, how do you explainto your family?" Around 63\%explained acne as being puberty related, $28 \%$ students explained it as being dirty face related, $26 \%$ studentsexplained as being climate related (summer), 23.1\% explained as food related and similarly, by use of many cosmetics (15.8\%), family history of acne in $23.9 \%$, sebaceous gland disorder (49.4\%) and dirty blood $(9.7 \%)$ (Table 4).

Similarly, an open question was asked regarding popular medication used in acne. They responded as topical clindamycin followed by oral antibiotics and herbal face wash. About 35\% did not know about the medicines used in the treatment of acne (Table 5).

Opinion about psychological impact:

The fourth part included questionnaire on psychological effect of acne intheir medical career. The effects included aggression (8.1\%), poor performance in study (13.4\%), depression (13.8\%), anxiety (15.8\%), impaired social contact $(16.2 \%)$, lack of confidence (31.2\%) and embarrassment (45.3\%). The impact of acne on opposite sex relation was found in $55.5 \%$, and interference in family relation due to acne was found in $11.1 \%$. Around $40 \%$ students considered that there was a bias in practical examination. History of suicidal tendency was present in 3.6\% (Table 6).

Table 1: Demographic Data

\begin{tabular}{|l|c|c|}
\hline Students & Frequency & Percent (\%) \\
\hline Male & 94 & 38 \\
Female & 153 & 61.9 \\
$>15$ years & 122 & 49.4 \\
15-20 years & 119 & 48.2 \\
20-25 years & 6 & 2.4 \\
\hline Total & $\mathbf{2 4 7}$ & $\mathbf{1 0 0 . 0}$ \\
\hline
\end{tabular}


Aryal E, et al. Psychological impact of acne vulgaris in basic science medical students

Table 2: Causative Factors for Acne.

\begin{tabular}{|l|c|c|}
\hline Causative factors for acne & Frequency & Percent (\%) \\
\hline Contagious & 7 & 2.8 \\
Bad Thought & 15 & 6.1 \\
Frequent washing of face & 26 & 10.5 \\
Family History & 26 & 10.5 \\
Heat/Summer & 53 & 21.5 \\
Cosmetic & 66 & 26.7 \\
Poor Hygiene & 82 & 33.2 \\
Diet & 96 & 38.9 \\
Puberty Related & 144 & 58.3 \\
Oily Face & 160 & 64.8 \\
\hline
\end{tabular}

Table 3: Preferred means of treatment

\begin{tabular}{|l|c|c|}
\hline Preferred means of treatment & Frequency & Percent (\%) \\
\hline None & 46 & 18.6 \\
\hline Herbal & 73 & 29.6 \\
\hline Beautician/saloon & 14 & 5.7 \\
\hline Ayurvedic & 55 & 22.3 \\
\hline Homeopathic & 19 & 7.7 \\
\hline Allopathic & 40 & 16.2 \\
\hline Total & 247 & 100.0 \\
\hline
\end{tabular}

Table 4: Terminologies used to explain about acne to the family members.

\begin{tabular}{|l|c|c|}
\hline Terminology & Frequency & Percent (\%) \\
\hline Puberty Related & 155 & 63 \\
\hline Dirty Face & 70 & 28.3 \\
\hline Heat/Summer & 64 & 26 \\
\hline Food & 57 & 23.1 \\
\hline Cosmetic uses & 39 & 15.8 \\
\hline Family History of Pimples & 59 & 23.9 \\
\hline Sebaceous Gland disease & 122 & 49.4 \\
\hline Dirty Blood & 24 & 9.7 \\
\hline
\end{tabular}

Table 5: Most common / popular acne medications.

\begin{tabular}{|l|c|c|}
\hline Most common medication & Frequency & Valid Percent \\
\hline None & 86 & 34.8 \\
\hline Oral antibiotics & 24 & 9.7 \\
\hline Clindamycin & 30 & 12.1 \\
\hline Isotretinion & 10 & 4.0 \\
\hline Healthy food & 5 & 2.0 \\
\hline Blood purifier & 8 & 3.2 \\
\hline Home Remedy & 9 & 3.6 \\
\hline Tretinion & 9 & 3.6 \\
\hline Herbal face wash & 23 & 9.3 \\
\hline Vitamin A & 13 & 5.3 \\
\hline Rose water & 7 & 2.8 \\
\hline Ayurvedic & 5 & 2.0 \\
\hline Estrogen hormone therapy & 3 & 1.2 \\
\hline Benzoyl peroxide & 15 & 6.1 \\
\hline Total & $\mathbf{2 4 7}$ & $\mathbf{1 0 0 . 0}$ \\
\hline
\end{tabular}


Table 6: Psychological Impact of acne.

\begin{tabular}{|l|c|c|}
\hline Psychological impact of Acne & Frequency & Percentage \\
\hline Embarrassment & 112 & 45.3 \\
\hline Anxiety & 39 & 15.8 \\
\hline Depression & 34 & 13.8 \\
\hline Aggression & 20 & 8.1 \\
\hline Lack of confidence & 77 & 31.2 \\
\hline Impaired social contact & 40 & 16.2 \\
\hline Poor performance in study & 33 & 13.4 \\
\hline Suicidal tendency & 9 & 3.6 \\
\hline Interference with opposite sex relationship & 137 & 55.5 \\
\hline Interference with family relationship & 29 & 11.7 \\
\hline Bias in practical exam & 97 & 39.3 \\
\hline
\end{tabular}

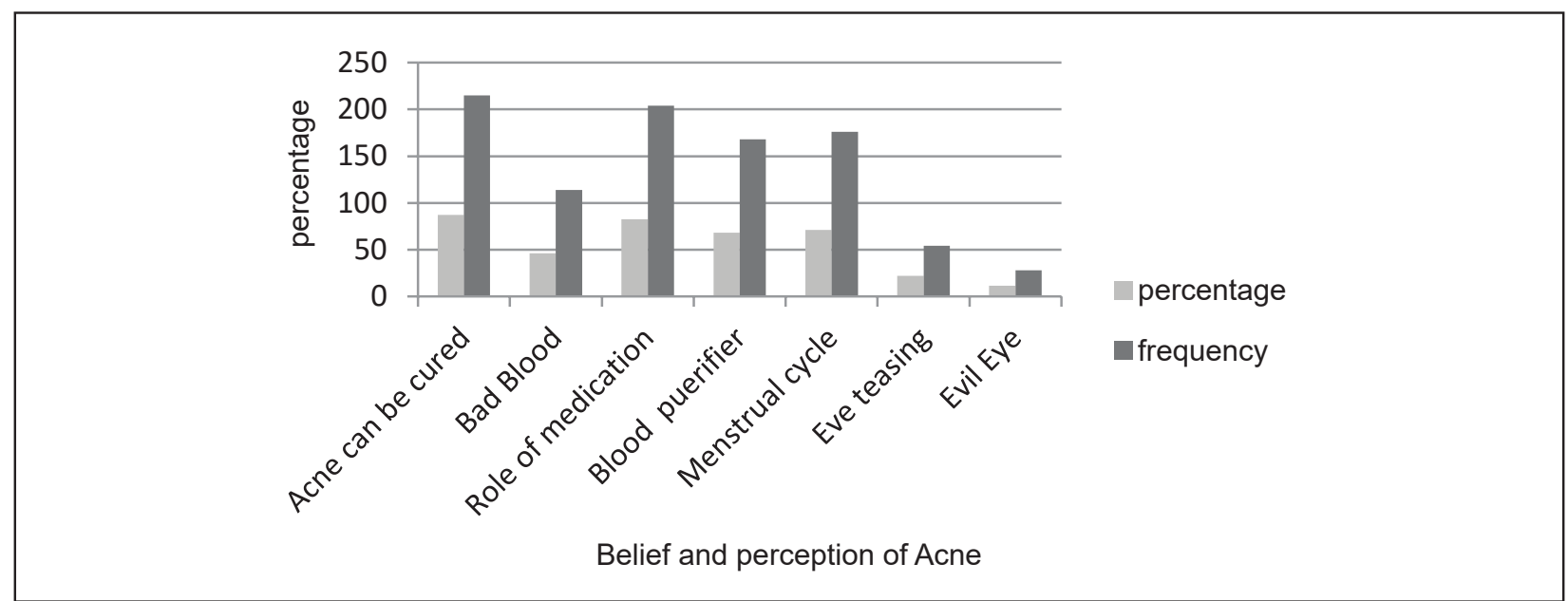

Figure 1: Belief and perception of acne.

\section{Discussion}

Acne vulgaris is a very common chronic skin disease present in adolescents that continue seven after twenties. Acne does not cause physical disability, but it frequently disfigures the patient if not treated in early phase. ${ }^{2}$ Ultimately, the psychosocial and occupational consequence of disfigurement is much more relevant than pure physical limitation. ${ }^{1}$ The knowledge about acne is still inadequate with a lot of misconceptions and myth. Thus, we studied the perception, knowledge and psychosocial impact of acne in basic science medical students.

In our study, we found that acne was more common in females (62\%) as compared to males (38\%). A similar study done in protuguese medical students revealed more females than male. ${ }^{2}$ The most common causative factor in our study was considered to be oily skin followed by puberty related condition, stress and diet. In a study by Al-Hoqail IA, 79\% of acne patients believed that acne was related to diet. $^{3}$ Similarly, in a study by
Poli F et al, diet was thought to exacerbate acne. ${ }^{4}$ Green $\mathrm{J}$ and colleague in their study on final year medical graduates of University of Melbourne, Australia found the same result but in lower frequency. ${ }^{5}$ Similar to the curriculum of $\mathrm{KU}$, dermatology is taught only in final year in Melbourne University, where the student of basic science has least knowledge about acne. In our study, $26.7 \%$ believed acne to be related withthe use of cosmetics where as Poli $\mathrm{F}$ et al reported that $58 \%$ believed the same. Acne is a common skin condition and treatment is recommended as risk of scarring is minimized if treated earlier. The best way to treat acne was $31.2 \%$ by herbal or home remedy in our sample whereas study done in Pakistan among undergraduate medical student, they preferred steroid and antibiotic cream.

An open question was asked about the most common medication they had heard about acne. Around 68\% believed that blood purifier would improve acne, around $35 \%$ sample answered as none and $12 \%$ answered as clindamycin. 
Misconceptions about acne are prevalent not only among adolescence and young adults and acne patients but also among family physician. ${ }^{6}$ Explanation of acne as a medical student to family members was as puberty related by $62.8 \%$ and as sebaceous gland disorder by $49.4 \%$.

Regarding the psychological impact of acne, $45.3 \%$ felt embarrassment followed by lack of confidence in $31.2 \%$, depression was highly thought to be a possible consequence of acne in $13.8 \%$ and impaired social contact in $16.2 \%$. Likewise, in Riyadh, more than half of the studied groups indicated that acne had an impact on loss of self-confidence. ${ }^{3}$ In the same study by AlHoqail IA, most of the patients (73\%) clarified that acne had no or minimal effect on their relationship which was similar to our study (55.5\%). ${ }^{3}$ A Nigerian study accomplished in 2009 summarized that only a small number reported that acne severely affected their relationship with their ability to make friends, relation with family member, which was similar to our study. ${ }^{7}$ Around $4 \%$ of our students thought that acne could lead to suicidal attempts, whereas, in a study done in Jeddah, Saudi Arabia, it was $17 \%$ which was much higher as compared to our study. ${ }^{3}$

\section{Limitations}

1) Study sample was less, only two medical college students were enrolled. 2) In the questionnaire, there were multiple responses for the same questions.

\section{Conclusion}

Basic science medical students may have impact directly, as those having acne themselves will suffer and secondly, they may disseminate the misconception in the community. Therefore, a health education program is needed from the community level and in all schools from secondary level. This may be achieved by organizing lectures or seminar at school along with a short-talked program in media including magazine, television, and radio. Problem Based Learning (PBL) including common skin disease should be discussed individually to all undergraduate medical students during basic science, as they play akey role in disseminating their knowledge in the community.

Financial disclosure: None.

Conflict of interest to disclosure: Non declared.

\section{References}

1. Farid-ur-Rehman, NiaziNAK. Beliefsand perceptions about acne among undergraduate medical students. JPAD. 2007;17(4):231-4.

2. Goncalves G, Amado JM, Matos ME, Massa A. The prevalence of acne among a group of Portuguese medical students. J EurAcadDermatolVenereol.2012;26(4):514-7. https://doi.org/10.1111/j.1468 3083.2011.04080.x

3. Al-Hoqail IA. Knowledge, beliefs and perception of youth towards acne vulgaris. Saudi Med J.2003;24(7):765-8.

4. Poli F, Auffret N, Beylot C, Chivot M, Faure $M$, Moyse $D$, et al. Acne as seen by adolescent: result of questionnaire study in 852 French

individuals. Acta DermVenereol. 2011;91(5):5316.https://doi.org/10.2340/00015555-1125

5. Green J, Sinclair RD. Perception of acne vulgaris in final year medical student written examination answers. Australas J Dermatol. 2001;42:98-101.https://doi.org/10.1046/j.14400960.2001.00489.x

6. Brajac I, Bilić-Zulle L, Tkalcić M, Loncarek K, Gruber F. Acne vulgaris: myths and misconceptions among patients and family physicians. Patient EducCouns. 2004;54:21-5.https://doi. org/10.1016/S0738-3991(03)00168-X

7. H Yahya. Acne vulgaris in Nigerian adolescentsprevalence, severity, beliefs, perception and practice. Int J Dermatol. 2009;48(5):498505.https://doi.org/10.1111/j.13654632.2009.03922.x 